\title{
LE STATUT COMMUNAUTAIRE DE LA POLYNÉSIE FRANÇAISE
}

\author{
Charles-Etienne Gudin*
}

From the point of view of France, French Polynesia is an integral part of the French Republic but it does have a certain degree of political autonomy. From the point of view of European Community law, French Polynesia has a special status under the Part 4 of the EC Treaty. That Part confers on French Polynesia the benefit of a special relationship with the European Union. In addition French law considers that those living overseas are all French citizens and therefore have under the EC Treaty the status of European citizen. In this paper the author considers the nature of the association of the French territories to the European Union that is provided in the Treaty and notes that it is not radically different from that provided in the Cotonou Agreement. Working from that point the article investigates whether the provisions of Part 4 alone apply to the overseas territories and seeks to identify the true range of the application of community law to the overseas territories.

Pour la France, la Polynésie française (PTOM) fait partie intégrante de la République mais possède une autonomie politique certaine.

En droit communautaire, la Polynésie française possède un statut particulier au sens de la quatrième partie du traité, qui lui confère le bénéfice de "relations particulières » avec l'Union Européenne,

Par ailleurs, le droit français considérant que les ressortissants d'outre-mer ont tous la qualité de citoyens français de telle sorte qu'ils ont au regard du traité CE la possession de la qualité de citoyen de l'Union européenne.

L'auteur explique que le contenu du régime d'association que la Communauté a prévue pour les PTOM n'est pas radicalement différent de celui que l'on peut trouver dans la convention de Cotonou et partant il s'interroge sur le point de savoir si seules les dispositions du traité figurant dans sa quatrième partie, sont applicables aux PTOM, et ce faisant tente de délimiter le champ d'application réel du droit communautaire aux PTOM.

* Directeur de la Revue des Affaires Européennes, Avocat au Barreau de Bordeaux. 
En droit interne la Polynésie française est une collectivité d'outre-mer dotée de l'autonomie au titre de l'article 74 de la constitution ${ }^{1}$. En droit communautaire, si la Polynésie française possède un statut particulier où elle est considérée comme un «pays ou territoire d'outre-mer» au sens de la quatrième partie du traité qui est spécialement consacrée à de telles entités, elle est aujourd'hui expréssément visée par l'annexe IV du traité qui donne une liste de ces «pays et territoires» géographiquement non européens, mais qui possèdent avec certains Etats membres de la Communauté ce que le traité appelle des «relations particulières». Derrière cet euphémisme se cache en fait le reflet d'une situation historique que n'ont connu que certains Etats membres et non pas les autres, selon qu'ils possèdent ou non une histoire coloniale et donc post coloniale commune avec ces pays et territoires dits «d'outre-mer». La plupart des «territoires d'outre-mer» de la France sont aujourd'hui indépendants et sont aujourd'hui partie à la convention de Cotonou.

Le second élément qui importe au regard du statut communautaire d'un tel pays ou territoire est son statut constitutionnel en droit interne. Pour la France, la Polynésie française fait partie intégrante de la République mais possède comme on le sait une autonomie politique certaine. Enfin, le droit français considère que les ressortissants d'outre-mer ont tous la qualité de citoyens français ce qui entraîne de jure au regard du traité CE la possession de la qualité de citoyen de l'Union européenne. C'est ainsi que pour la France le tribunal administratif de Papeete est bien une juridiction française ce qui lui confère au regard du droit communautaire, la qualité de juridiction d'un Etat membre habilitée à ce titre à poser à la Cour de justice des questions préjudicielles notamment sur l'interprétation du droit communautaire. En revanche si la situation peut varier entre un PTOM et un autre au regard de cette qualification de «juridiction d'un Etat membre», c'est au seul droit communautaire de décider jusqu'où il est susceptible de s'appliquer dans un pays ou territoire d'outre-mer ${ }^{2}$. On notera enfin qu'à la différence des régions ultrapériphériques - catégorie à laquelle appartiennent les départements français d'outre-mer, les PTOM ne font pas partie du territoire communautaire.

Comme on le verra, le contenu du régime d'association que la Communauté a prévue pour les PTOM n'est pas radicalement différent de celui que l'on peut trouver dans la convention de Cotonou par exemple qui regroupe comme on le sait les 70 Etats indépendants d'Afrique de Caraïbe et du Pacifique. Le statut de la Polynésie est aujourd'hui couvert par une décision d'association datant de

1 Le statut d'autonomie a été conféré à la Polynésie française par une loi du 6 septembre 1984. Il a déjà permis au territoire de s'administrer plus librement. Par la suite, ce statut a été modifié par une loi du 12 juillet 1990 qui renforce les attributions du gouvernement du Territoire. Mais c'est en 1995 et surtout en 1996 qu'un nouveau statut est adopté afin de conforter cette autonomie, d'améliorer le fonctionnement des institutions polynésiennes et d'étendre les compétences territoriales. Le statut actuel de la Polynésie résulte de la loi organique $\mathrm{n}^{\circ}$ 2004-192 du 27 janvier 2004 portant statut d'autonomie de la Polynésie française. Il s'agit véritablement de la première application de la révision opérée par la loi constitutionnelle $n^{\circ} 2003-276$ relative à l'organisation décentralisée de la République.

2 CJCE. 12 décembre 1990, Kaefer et Procacci aff C-100/89 et C-101/89 et C-101/89, Rec 1990, I, 4647. 
2001, relative à l'association des PTOM à la $\mathrm{CEE}^{3}$. Compte tenu de l'évolution que connaît aujourd'hui le statut des pays ACP la similitude de ces deux régimes tend aujourd'hui à s'estomper.

Comme on le verra, il n'est pas facile de déterminer avec précision le champ d'application du droit communautaire aux PTOM car l'article 299 s'il vise bien cette catégorie se contente d'opérer un renvoi aux dispositions de la quatrième partie du traité. Cela signifie-t-il pour autant que seules les dispositions du traité figurant dans la quatrième partie du traité sont applicables aux PTOM? Ce n'est pas notre avis ainsi que celui d'une partie de la doctrine pour qui il conviendrait de distinguer deux catégories de règles en dehors même des règles de procédure: celles qui ont trait à l'établissement de l'union douanière et du marché intérieur de la Communauté qu'accompagnent des politiques et des actions communautaires et celles qui constituent des dispositions comme la première partie du traité (Les principes), la deuxième (la citoyenneté), la cinquième (Les institutions de la Communauté) et la sixième (les dispositions générales et finales). Mais rien ne permet d'affirmer avec précision l'existence d'une telle distinction. Ce qui est certain est que d'autres parties du traité sont susceptibles de s'appliquer face à une situation mettant en jeu les PTOM. On ne citera que deux exemples, la possibilité reconnue au Conseil de réduire un avantage accordé au titre de l'association des PTOM au vu d'un risque de désorganisation de l'organisation commune du marché d'un produit agricole. La question s'est posée concrètement pour des importations massives de riz dans la Communauté. L'autre exemple réside dans l'émergence de la citoyenneté de l'Union. Il est évident que quels que soient les pouvoirs que l'on peut encore reconnaître aux Etats membres dans l'organisation de l'exercice de tels droits, une porte s'ouvre ainsi aux Polynésiens par exemple en tant que citoyens à part entière de l'Union européenne.

\section{LE STATUT D'ASSOCIATION À LA COMMUNAUTÉ EUROPÉENNE}

La Polynésie française est apparue en droit communautaire dans les bagages de la France. C'est en effet à celle-ci que l'on doit l'élaboration dans le traité CEE d'un statut particulier d'association pour une catégorie de territoires dits «d'outre-mer» selon une terminologie aujourd'hui un peu datée. C'est à la conférence intergouvernementale de Venise qui s'est tenue les 20 et 30 mai 1956 que le principe en a été posé afin de pouvoir faire bénéficier des avantages de la construction communautaire ces pays et territoires qui bien que non européens d'un point de vue géographique avaient du fait même de l'histoire, voire tout simplement de l'histoire coloniale, conservés des liens dits «particuliers» selon la terminologie du traité avec un Etat membre. Mais comme le traité CEE est fondé sur l'idée même de la constitution d'un «marché commun», c'est-à-dire de la suppression progressive entre les Etats membres de toute barrière tarifaire et non tarifaire accompagnée notamment de règles de concurrence, il n'était pas certain qu'un tel régime fut en lui-même adapté aux besoins de tels pays et territoires dont le principal souci était bien évidemment celui de leur développement économique et social, mais aussi l'éloignement, l'insularité, leur faible superficie

3 JO 2001 L 314 du 30 novembre 2001, rectif. JO 2001 L 324 du 7 décembre 2001. 
parfois, à moins qu'ils ne soient en même temps largement dispersés sur des espaces immenses. Le relief, le climat autant de facteurs souvent contraignants sans oublier la dépendance bien souvent à l'égard d'un petit nombre de produits de base. Il y avait donc pour ces différents pays et territoires d'outre-mer des impératifs autrement plus urgents que de constituer un marché intérieur, commun à l'ensemble des Etats membres.

Mais comment faire bénéficier ces pays des avantages que pouvait leur procurer le fait que les européens étaient en voie d'intégration économique et indirectement politique avec les besoins déclarés de pouvoir atteindre un meilleur niveau de développement? Il est évident qu'avant cette évolution de l'intégration communautaire des Etats européens, c'était la France qui entretenait avec la Polynésie des rapports qui ont évolué avec le temps. Le rapport de la Polynésie française à la Communauté européenne n'est donc dans une certaine mesure qu'une conséquence de ces rapports bilatéraux particuliers avec un Etat membre. Mais ce serait, à nos yeux une grave erreur que de considérer que le droit communautaire n'ouvre pas également à la Polynésie des perspectives nouvelles non seulement économiques en permettant l'adaptation à son profit de certaines règles du commerce international par exemple, mais également au regard de la notion de citoyen européen ou plus largement dans la possibilité de dialoguer avec l'autorité communautaire sans être systématiquement représentée par la France elle-même. La Polynésie acquiert elle au moyen du droit communautaire une visibilité nouvelle? C'est là nous semble-t-il une idée intéressante qu'il convient d'approfondir.

\section{A La Nature du Régime d'association}

L'idée de départ est de faire bénéficier les pays et territoires d'outre-mer des mesures préférentielles que les Etats membres des Communautés européennes s'accordent réciproquement. Le régime des échanges entre la Communauté et ces pays et territoires, allait ainsi pouvoir faire l'objet de décisions particulières des Etats membres réunis au sein du Conseil. Mais on a franchi un pas de plus en intégrant ensuite ce régime dans le corps du traité lui-même afin de pouvoir mieux en définir les contours généraux et de préciser les règles de fonctionnement du Conseil dans son travail d'élaboration des règles de droit dérivé qui seraient prises sur la base de ces principes et ce mécanisme de décision communautaire.

C'est ainsi que le $1^{\text {er }}$ janvier 1958, le traité CEE contenait à la fois des règles de fond et des règles de procédure spécialement conçues pour ce type de pays et de territoires pour lesquels on envisageait ainsi un régime spécial d'association à la Communauté. Les établissements français de l'Océanie figuraient parmi les bénéficiaires en tant que territoires sous tutelle française.

Une liste des Pays et Territoires d'Outre-mer fut élaborée par les Etats membres et annexée au traité CEE (annexe IV à l'origine). Cette liste a ensuite été complétée à plusieurs reprises. On notera que le régime d'association à la Communauté ainsi prévu ne vise pas d'autres territoires non européens également liés avec certains Etats membres, comme par exemple les Départements française d'Outre-mer. C'est que le lien qui unit ces territoires aux Etats membres concernés n'est pas 
de même nature. Ils sont considérés par ces Etats comme suffisamment intégrés pour quils n'apparaissent dans l'ordre juridique communautaire qu'à travers la personne juridique de ces Etats eux-mêmes. C'est donc bien du droit national de chaque Etat membre et plus particulièrement de son droit constitutionnel que dépend la qualification communautaire de PTOM. Il appartient ensuite aux Etats membres de faire figurer ces pays ou territoires dans la liste prévue à cet effet. En pratique le statut spécial que prévoit le traité en faveur des PTOM ne s'appliquera qu'aux entités qui figurent expréssément dans la liste prévue à cet effet.

C'est ainsi que la Polynésie française apparait bien sur la liste de pays et territoires ayant des relations particulières avec certains Etats membres de la Communauté. Il s'agit d'après la liste actuellement en vigueur, des «Territoires d'outre-mer de la république française». Située comme on le sait au milieu du Pacifique, la Polynésie française est relativement peuplée par rapport à d'autres territoires et pays d'outre-mer figurant sur cette liste. Si la surface terrestre est relativement modeste, celle de son territoire maritime est en revanche très importante. La Polynésie dispose par ailleurs d'après le droit de la mer, d'une importante zone économique exclusive. Dans l'ensemble des PTOM concernés, elle apparaît comme un ensemble relativement développé. Il n'en reste pas moins vrai que l'éloignement géographique de l'Europe, l'étroitesse du marché local et d'autres facteurs qui lui sont propres constituent toujours un handicap pour son développement économique et social. Il en résulte que sa balance commerciale est souvent déficitaire et que les échanges se font essentiellement avec la France dont l'appui reste très important.

\section{Un régime particulier que prévoit le traité $C E$}

Pour comprendre comment le traité CE s'applique à la Polynésie française, il est nécessaire de se rapporter à l'article 299 du traité qui détermine son champ d'application, puis de se rapporter à la quatrième partie du traité relative au régime d'association à la Communauté des pays et territoires d'outre-mer.

(a) Le champ d'application du traité $\mathrm{CE}$ à la Polynésie française

L'article 299 détermine le champ d'application du traité. Trois situations nous intéressent ici directement: Le régime général selon lequel, le traité CE s'applique d'abord à ses Etats membres, le régime des «départements français d'outre-mer» et plus généralement des pays et territoires intégrés à un Etat membre en particulier et enfin celui qui nous concerne et que vise le paragraphe 3, alinéa 1, de l'article 299. Selon cette disposition: «Les pays et territoires d'outre-mer dont la liste figure à l'annexe II du présent traité font l'objet du régime spécial d'association défini dans la quatrième partie de ce traité»».

On remarquera que cet article ne donne pas de définition des PTOM. On se rapportera cependant à la définition générale qui figure cependant à l'article 182 du traité CE. Il s'agit des «pays et territoires non européens entretenant avec le Danemark, la France, les Pays-Bas et le RoyaumeUni des relations particulières». En outre, il est précisé que «Ces pays et territoires, ci après 
dénommés «pays et territoires» sont énumérés à la liste qui fait l'objet de l'annexe II du présent traité».

On comprendra que l'article 299 du traité afin de déterminer son champ d'application fait implicitement un renvoi aux dispositions constitutionnelles de chacun des Etats membres dont il reprend l'appellation officielle sans se référer jamais à ses composantes géographiques. La «République française» est ainsi visée en tant que telle. C'est dans l'arrêt Hansen ${ }^{4}$ que la Cour de Justice des Communautés a eu l'occasion de préciser dans quelles conditions le traité s'appliquait à des territoires géographiquement non européens. Pour la France, comme pour la Cour qui avait repris à son compte son argumentation, le statut communautaire des entités concernées est d'abord défini par référence à la constitution de l'Etat membre avec lequel il existe de telles relations particulières. C'est ainsi que la Cour a décidé que les dispositions générales du traité ainsi que le droit dérivé devaient s'appliquer purement et simplement aux départements d'outre-mer dans la mesure où ils font partie intégrante de la République française.

Mais l'affirmation d'un tel principe n'est pas incompatible avec la possibilité toujours offerte par le traité et sous certaines conditions qu'il définit de pouvoir faire bénéficier ces entités non européenne intégrées à l'un des Etats membres de mesures communautaires spécifiques en vue de répondre à leurs besoins. On retrouve ainsi la formule de l'article 299, paragraphe 2 du traité CE qui se rapporte aujourd'hui explicitement aux «Départements français d'outre-mer». Le premier alinéa précise que «Les dispositions du présent traité sont applicables aux départements français d'outremer (...)» alors que l'alinéa suivant prévoit la possibilité pour le Conseil statuant à la majorité qualifiée des Etats membres de prendre en effet des mesures spécifiques en faveur des ces entités intégrées. Il n'en reste pas moins que pour le droit constitutionnel français, la nature juridique de la Polynésie est d'être un «Pays d'outre-mer au sein de la République». Il n'en reste pas moins que la constitution française garantit dans le même temps son autonomie.

C'est donc bien de l'intégration ou non dans l'ordre juridique d'un Etat membre que dépend l'application du droit communautaire à ce type d'entités géographiquement non européennes mais entretenant avec certains Etats membres des relations que le traité qualifie de «particulières». La réponse à cette question préalable relative au caractère intégré ou non d'une telle entité, dépend bien évidemment du droit constitutionnel interne de chaque Etat membre concerné. De ce point de vue, le droit communautaire ne fait que prendre acte d'une situation politique donnée qui relève du seul droit interne.

Peut-on à l'inverse se contenter de dire que si le traité CE est ainsi applicable à la «République française» comprenant les entités qui lui sont intégrées comme ce que le traité CE qualifie encore de «départements français d'outre-mer», à l'inverse, il ne trouve pas à s'appliquer à une entité comme la

4 CJCE, 10 octobre 1978, Hansen, aff 148/77: Rec 1978,1787. 
Polynésie française qui ne constitue ni un territoire européen de la République, ni un département d'outre-mer.

La première réponse est purement formelle: le traité CE est applicable à la Polynésie française dans la mesure et dans la mesure seulement où elle constitue une catégorie juridique relevant de l'application de la quatrième partie du traité CE (en ce qu'elle constitue un PTOM).

Mais cette réponse n'est pas juridiquement très satisfaisante, parce qu'elle se fonde sur de simples déclarations des Etats membres sans que l'on puisse ainsi connaître quels sont objectivement les critères d'une telle application du traité. La doctrine considère qu'il peut exister des situations dans lesquelles il ne s'agit ni d'un territoire européen d'un Etat membre, ni d'un territoire non européen intégré par le droit constitutionnel interne, ni d'un PTOM au sens de l'article 182 du traité CE. Dans ce cas, l'article 299 du traité visant à une application graduée du droit communautaire semble permettre d'exclure de l'application du droit communautaire des entités géographiques qui ne correspondrait à aucune des catégories prévues par le traité.

Cette question est pour la Polynésie française purement doctrinale, puisqu'il ne fait aucun doute en droit positif que la Polynésie française relève de l'application du droit communautaire spécial que constituent les dispositions de la quatrième partie du traité et des décisions prises à l'unanimité pour son application par les Etats membres réunis au sein du Conseil. C'est ainsi que le président de la commission dans une réponse à un parlementaire européen avait indiqué que les PTOM limitativement énumérés dans l'annexe IV du traité CEE «ne font l'objet que du régime spécial d'association défini dans la quatrième partie du traité». ${ }^{5}$

(b) Le régime d'association de la Polynésie française

Le régime d'association à la Communauté que vise l'article 299 du traité CE figure dans la quatrième partie du traité qui est spécialement consacrée à cette question. Est-ce une politique communautaire? A strictement parler, il ne s'agit pas d'une politique au sens où elles figurent dans la troisième partie du traité CE. La libre circulation des marchandises, l'agriculture, la libre circulation des personnes, des services et des capitaux, les visas, l'asile, l'immigration et les autres politiques liées à la libre circulation des personnes, les transports, les règles communes sur la concurrence, la fiscalité et le rapprochement des législations, la politique économique et monétaire, l'emploi, la politique commerciale commune, la coopération douanière, la politique sociale, l'éducation, la formation professionnelle et la jeunesse, la culture, la santé publique, la protection des consommateurs, l'industrie, la cohésion économique et sociale, la recherche et le développement technologique, l'environnement, la coopération au développement, la coopération économique, financière et technique avec les pays tiers, sont considérées au sens large comme «Les politiques de la Communauté», comme l'indique le titre de la troisième partie du traité qui leur est consacrée.

5 Réponse du président de la Commission à la question écrite de M Glinne, député européen, relative au champ d'application territorial du droit communautaire. 
Non, «L'association des pays et territoires d'outre-mer», n'est pas à proprement parler une politique de la Communauté à supposer que l'on puisse définir cette notion. C'est davantage nous semble-t-il une certaine manière d'appliquer le droit communautaire aux pays et territoires d'outre-mer que vise le traité et dont la liste figure dans son annexe.

Si tel est bien le cas, que signifie globalement un tel régime? Il faut s'interroger ici sur la signification et la valeur juridique des décisions qui vont pouvoir être prises par les Etats membres réunis au sein du Conseil, qui à l'unanimité comme le prévoit l'article 187 du traité CE, vont pouvoir établir des dispositions relatives aux modalités et à la procédure de l'association. D'une manière générale les grandes lignes du régime d'association figurent dans les articles 182 à 188 qui constituent la quatrième partie du traité CE.

L'article 182 pose le principe de l'association des pays et territoires non européens entretenant avec certains Etats membres des relations particulières (alinéa 1); il définit le but de cette association qui est «la promotion du développement économique et social des pays et territoires» d'une part et corrélativement «l'établissement de relations économiques étroites entre eux et la Communauté dans son ensemble» (alinéa 2). Enfin il est indiqué que «L'association doit en premier lieu permettre de favoriser les intérêts des habitants de ces pays et territoires et leur prospérité, de manière à les conduire au développement économique, social et culturel qu'ils attendent» (alinéa 3). Cette dernière précision n'est pas sans intérêt, car elle indique bien implicitement que les règles du traité CE qui sont en principe destinées à favoriser la réalisation d'objectifs communautaire vont ici servir dans la mesure du possible un autre but qui est le développement de ces territoires non européens qui méritent aux yeux de l'ensemble des Etats membres un traitement particulier (expréssément mentionné et développé dans le traité) parce que certains d'entre eux ont de liens particuliers ( c'est-à-dire à l'origine en fait une histoire coloniale puis post coloniale) avec de tels pays ou de tels territoires que rien a priori ne destinent à être concernés par le processus d'intégration européenne. De ce point de vue l'action communautaire prévue en leur faveur ne fait que relayer et communautariser de actions unilatérales menées par les Etats membres concernés visà-vis de ces entités dont ils avaient en quelque sorte la responsabilité.

L'article 183 va donner une liste des objectifs que poursuit l'association. C'est en quelque sorte l'objet même de l'association des ces pays et territoires à la Communauté. C'est également le contenu explicite d'un tel régime que le traité CE prévoit en leur faveur. Tout d'abord, il impose une obligation aux Etats membres de la Communauté. Celle d'appliquer à leurs échanges commerciaux avec ces pays et territoires, le régime qu'ils s'accordent entre eux dans les échanges (paragraphe 1). Inversement, il impose une obligation générale aux PTOM. Celle d'appliquer dans leurs échanges commerciaux avec les Etats membres, comme dans ceux qu'ils pratiquent avec d'autres PTOM le même régime que celui qui régit les relations particulières qu'il entretient avec l'Etat membre dont il dépend encore relativement ( par exemple, la France pour la Polynésie) (paragraphe 2). Par ailleurs il est indiqué que ce sont désormais les Etats membres dans leur ensemble «les Etats membres» qui vont contribuer dans le cadre de ce régime d'association aux investissements réalisés 
dans les pays ou territoires en question (paragraphe 3). Enfin, pour les investissements financés par la Communauté, il est indiqué que la concurrence doit être ouverte à l'ensemble des investisseurs communautaires qui pourraient être intéressés par les opérations envisagées (paragraphe 4). Pour finir, l'article 183 vise le droit d'établissement des ressortissants et des sociétés. Il est indiqué que les règles du traité CE s'y appliquent sous réserve des règles particulières qui pourront être adoptées à l'unanimité par les Etats membres réunis au sein du Conseil comme le prévoit expréssément l'article 187. Ce sont donc les décisions du Conseil prises dans le cadre de la procédure de l'article 187 qui primeront le cas échéant le droit communautaire général.

En d'autres termes, il s'agit de la situation inverse de la solution généralement retenue où le droit dérivé communautaire doit nécessairement respecter la règle dont il est issu. En fait ce n'est qu'une contradiction apparente, car la règle matérielle dont est issue une décision prise au titre de l'article 187 du traité CE est constituée exclusivement des dispositions correspondantes des article 182 à 188 du traité (lex specialis) et lorsqu'elles ne prévoient rien de particulier, elles autorisent le Conseil a prendre des décisions qui différent et dérogent le cas échéant de celles qui figurent dans les politiques de la Communauté qui ne s'appliquent donc pas directement aux PTOM.

L'article 184 est relatif au régime douanier des importations originaires des pays et territoires d'outre-mer lors de leur entrée dans les Etats membres de la Communauté (paragraphe 1), du régime douanier applicable par les PTOM à l'entrée des marchandises originaires des Etats membres ou des autres pays et territoires d'outre-mer (paragraphe 2), d'une exception prévue en faveur des PTOM leur permettant de percevoir dans certaines conditions des droits de douane en leur faveur lors de l'importation des produits en provenance des Etats membres ou d'autres PTOM (paragraphe 3), de règles propres à certains pays et territoires en braisons d'obligations douanières internationales particulières, l'interdiction enfin de toute forme de discrimination dans l'établissement ou la modification des tarifs douaniers (paragraphe 5).

L'article 185 vise la possibilité pour un Etat membre de demander des mesures spéciales en cas de détournement de trafic (article 185).

L'article 186 vise la question de la libre circulation des travailleurs.

L'article 187 est une disposition de nature institutionnelle qui confie au Conseil (C'est-à-dire aux Etats membres réunis au sein du Conseil) de prendre des décisions d'application des principes figurant dans la quatrième partie du traité. Ainsi le Conseil a-t-il la possibilité de prendre des décisions (ce qui constitue un acte de nature communautaire) destinées aux PTOM et aux Etats membres, relatives aux modalités de mise en œuvre du régime d'association qu'il s'agisse de ces règles matérielles ou de ses règles de procédure. D'une manière générale, il est rappelé que les Etats membres dans le cadre d'une telle procédure doivent tenir compte des réalisations acquises. Il existe donc un effet de «lock step» en faveur des PTOM dans le cadre de ce que la Communauté peut faire pour eux. C'est une sorte d'acquis communautaire spécial aux PTOM que prévoir ainsi le traité.

L'article 188 ne concerne pas la Polynésie française. 


\section{L'objectif poursuivi par le régime d'association}

Quant à l'objectif que poursuit le régime d'association, il apparaît de manière générale à l'article 182, comme il apparaissait à l'article 299 pour les Départements français d'outre-mer notamment. C'est que concrètement les problèmes sont les mêmes qu'il s'agisse des DOM ou des PTOM pour ne reprendre que la terminologie communautaire. Il s'agit de problèmes de développement et des problèmes d'éloignement par rapport aux grands flux industriels et commerciaux qui caractérisent le continent européen et plus généralement le commerce mondial des produits et des services. Toute action de ce type a pour objectif d'éviter la marginalisation des territoires concernés que ce soit économiquement, socialement ou plus généralement culturelle. De ce point de vue, le régime d'association des PTOM à la Communauté a pour objectif essentiel d'essayer d'éviter autant que se peut une telle marginalisation.

\section{Une communautarisation des règles de fonds et de procédure qui est propre à ce régime}

On a vu précédemment quelles étaient les règles de fond et de procédure que les Etats membres ont préférés insérer dans le traité plutôt que de se contenter de passer des accords entre eux. Ce qui caractérise un tel régime est donc bien la communautarisation de l'action qui est menée en leur faveur. D'une simple coopération entre les Etats membres concernés et a fortiori d'un simple rapport unilatéral de type administratif et politique national, pour l'Etat membre directement concerné, on est insensiblement passé à une véritable intégration à la fois fonctionnelle et matérielle de ce type de «politique» au plan communautaire. Cette intégration implique donc des Etats membres qui n'ont aucun passé historique de cette nature ayant conduit à ce type de liens justifiant un tel régime d'association. Ainsi les relations communautaires avec les PTOM ont-elles le mérite de faire table rase d'un passé parfois difficile en terme identitaire. Le caractère «supranational» d'une telle action communautaire vient ici rejoindre des préoccupations parfois infra nationales en quête d'une plus grande autonomie. Ici comme là, la nation n'apparaît donc pas, au moins d'un point de vue juridique, comme un horizon insurpassable, pour paraphraser certains.

\section{B Les Effets du Régime d'association}

\section{$1 \quad$ L'adaptation des règles communautaires au statut de la Polynésie française}

Comment se passe concrètement l'adaptation des règles communautaires au statut des PTOM et plus particulièrement de la Polynésie française? Car c'est bien d'adaptation des règles communautaires qu'il s'agit et non pas comme le prétendent certains commentateurs, voire des responsables politiques communautaires d'une «non application du droit communautaire aux PTOM». Lorsque la Cour de justice parle de cette non application, elle vise en réalité, la non application du droit communautaire général. Cependant même vis-à-vis du droit communautaire général reste la question essentielle comme nous le verrons du statut de citoyen européen que détiennent les Polynésiens en tant qu'ils sont des nationaux d'un Etat membre. La question est ici loin d'être résolue, car chassé classiquement par la «petite porte» de la «politique» d'association des 
PTOM, le droit communautaire revient dans quelques uns de ses principes fondamentaux par «la grande porte» désormais de la citoyenneté européenne.

\section{La valeur relative des objectifs poursuivis par le traité CE en faveur des PTOM}

Les obligations résident notamment dans le fait de ne pouvoir imposer de droits de douane à l'entrée dans les Etats membres des marchandises originaires des PTOM. Ainsi lorsque des marchandises originaires du territoire de la Polynésie française entrent dans n'importe quel Etat membre de la Communauté, elles sont exemptées de droits de douane. Cependant lorsque le Conseil arrête des mesures au titre de l'article 187, deuxième alinéa, il doit tenir compte des principes figurant dans la quatrième partie du traité CE relatives au régime d'association en faveur des PTOM, mais également, le cas échéant d'autres principes du droit communautaire, comme ceux par exemple qui se rapportent à la politique agricole ${ }^{6}$. En fait ceci constitue bien la preuve nous semble-t-il que le droit communautaire s'applique en fait aux PTOM, le cas échéant en dehors du cadre strict de la quatrième partie du traité. Il peut arriver que le Conseil ait à trancher entre deux intérêts le cas échant contradictoires, celui des PTOM d'une part et celui de la Politique agricole, donc des Etats membres eux-mêmes d'autre part. On notera que dans ce cas, la Cour de Justice reconnaît au Conseil un large pouvoir d'appréciation. S'il est vrai que le Conseil se voit confier une mission particulière par l'article 187 en faveur des PTOM, il lui appartient également de veiller dans le cadre de la politique agricole (Voir le Titre II de la troisième partie du traité CE consacrée aux politiques de la Communauté), à ce que le marché de certains produits agricoles ne soit pas désorganisé par des importations massives et à des prix particulièrement compétitifs de certains produits similaires importés en franchise de droit d'un PTOM par ailleurs protégé par les dispositions de la quatrième partie du traité.

Le problème s'était posé pour le marché communautaire du riz ${ }^{7}$. Dans un tel cas, le Conseil peut, par dérogation au principe figurant à l'article 183, paragraphe 1 du traité CE (qui veut que les Etats membres appliquent normalement à leurs échanges commerciaux avec les pays et territoires d'outre-mer, le régime qu'ils s'accordent entre eux en vertu du traité) et par dérogation également aux règles figurant dans les décisions qu'il a pu prendre au titre de l'article 187 ( ses pouvoirs propres lorsque la Communauté agit en faveur des PTOM), être exceptionnellement amené à diminuer certains avantages précédemment accordés aux PTOM.

On constate à ce propos que les «réalisations acquises» par les PTOM dans le cadre de l'association avec la Communauté, ne sont pas absolues et que le Conseil peut toujours dans le cadre du large pouvoir d'appréciation qu'il possède faire des arbitrages entre plusieurs politiques communautaires qui entrent comme ici en concurrence les unes avec les autres et cela le cas échant

6 CJCE, 22 novembre 2001, Royaume des Pays-Bas contre Conseil de l'Union européenne, aff C-110/97, Rec. 2001, I-08763.

7 Affaire C-110/97 précitée supra n 6. 
au détriment des PTOM qu'il a cependant pour mission spécifique de protéger. Cet objectif est donc relatif et doit être envisagé de façon transversale vis-à-vis des autres actions que peut également mener la Communauté lorsqu'un conflit de cette nature se présente.

Dans ce cas, le juge communautaire, face à la reconnaissance de sa part d'un tel pouvoir d'appréciation de la part du Conseil se limitera à examiner dans le cadre d'un éventuel recours, si l'exercice de ces pouvoirs n'est pas entaché d'une erreur manifeste d'appréciation ou d'un détournement de pouvoir ou encore si les institutions communautaires n'ont pas manifestement dépassé les limites de leur pouvoir d'appréciation.

\section{Les mesures communautaires en faveur de la Polynésie française}

Parmi les mesures qui sont en faveur de la Polynésie française on citera celles qui lui permettent de percevoir des droits de douane et des taxes d'effet équivalent à des droits de douane, qu'ils aient ou non existé au moment de l'entrée en vigueur du traité CEE à condition que les droits ou les taxes qui ont été perçues à cette occasion correspondent aux nécessité du développement de la Polynésie telles que le traité et le droit dérivé le reconnaissent formellement. En effet, l'article 184, paragraphe 3 , alinéa 1 vise les conditions dans lesquelles les pays et territoires d'outre-mer peuvent percevoir des droits de douane. Il faut que ces droits ou taxes «répondent aux nécessité de leur développement et aux besoins de leur industrialisation». Il est également nécessaire si elles ont un caractère fiscal qu'elles servent effectivement à alimenter leur propre budget. On retrouve ainsi l'exigence que fixe l'article 182, alinéa 3: «Conformément aux principes énoncés dans le préambule du présent traité, l'association doit en premier lieu permettre de favoriser les intérêts des habitants de ces pays et territoires et leur prospérité, de manière à les conduire au développement économique, social et culturel qu'ils attendent».

L'article 3, paragraphe 1, lettre g) énonce en effet, qu' «Aux fins énoncées à l'article 2, l'action de la Communauté comporte, dans les conditions et selon les rythmes prévus par le présent traité:» (...) «l'association des pays et territoires d'outre-mer en vue d'accroître les échanges et de poursuivre en commun l'effort de développement économique et social». Il en résulte également qu'en se référant «aux fins énoncées à l'article 2», le développement harmonieux et durable des activités économiques, le niveau d'emploi et de protection sociale élevé, l'égalité entre les hommes et les femmes, une croissance durable et non inflationniste, un haut degré de compétitivité et de convergence des performances économiques, un niveau élevé de protection et d'amélioration de la qualité de l'environnement, le relèvement du niveau et de la qualité de la vie, la cohésion économique et sociale et la solidarité entre les Etats membres, pour être des «fins énoncées» à l'article 2 qui visent l'ensemble des Etats membres, n'en sont pas pour autant interdites aux PTOM qui cherchent au contraire à atteindre à terme un tel niveau de développement avec l'aide des Etats membres. Ce qui différents en revanche ce sont les instruments qui sont pour les Etats membres «l'établissement d'un marché commun, d'une union économique et monétaire et la mise en œuvre des politiques ou actions communes visées aux articles 3 et 4 ». Les PTOM simplement associés à la 
Communauté n'y participent pas directement, mais les Etats membres conviennent de les aider à combler le retard qui peut exister vis-à-vis d'autres objectifs communs.

4 La question de la libre circulation des professionnels

Dans une affaire relative à l'application de la convention d'association, la France a été amenée à soutenir que les directives communautaires relatives à la reconnaissance mutuelle des diplômes ne s'appliquaient pas aux PTOM. La première chose que l'on doit constater est que ces directives ne prévoient pas elles mêmes - si elles s'appliquent ou non aux pays et territoires relevant de l'application de la quatrième partie du traité. Mais la Cour n'a pas répondu dans cette affaire à cette question qui n'était pas indispensable à la solution du litige ${ }^{8}$.

\section{L'ÉVOLUTION DU STATUT COMMUNAUTAIRE DE LA POLYNÉSIE FRANÇAISE}

\section{A L'évolution du statut communautaire de la Polynésie au regard des développements récents du droit communautaire général}

\section{Le champ d'application matérielle du droit communautaire aux PTOM}

Dans une affaire relative à l'exercice par une personne résidant dans un PTOM de ses droits politiques aux élections au Parlement européen ${ }^{9}$, la Cour de justice des Communautés a considéré que les solutions qu'elle avait dégagée dans une affaire Bernard Leplat c/ Territoire de la Polynésie française sont toujours d'application. Elle a notamment rappelée que «les PTOM font l'objet d'un régime spécial d'association défini dans la quatrième partie du traité (articles $182 \mathrm{CE}$ à $188 \mathrm{CE}$ ), de sorte que les dispositions générales du traité ne leur sont pas applicables sans référence expresse». En affirmant ce principe, la Cour se fonde sur deux affaires plus anciennes, l'arrêt Leplat précité et l'affaire Pays-Bas Conseil.

On rappellera qu'au point 10 de l'arrêt Leplat, la Cour avait considéré qu':

Afin de répondre aux questions de la juridiction de renvoi, il convient de rappeler, à titre liminaire, la nature de l'association prévue par le traité CEE pour les pays et territoires d'outre-mer (ci après «PTOM»). Cette association fait l'objet d'un régime défini dans la quatrième partie du traité (articles 131 à 136) ${ }^{10}$, de sorte que les dispositions générales du traité ne sont pas applicables aux PTOM sans référence expresse. Aux termes de l'article 131, le but de cette association «est la promotion du développement économique et social des pays et territoires et l'établissement de relations économiques étroites ventre eux et la Communauté dans son ensemble». L'article 132 définit les objectifs de

8 CJCE, arrêt du 12 décembre 1990, aff C-236/88 Commission contre France. Rec. 1990, I, 4611 s.

9 CJCE, arrêt du 12 septembre 2006, M G Eman, O B Sevinger contre College van burgemeester en wethouders van den Haag. Voir infra point 2.

10 Devenus aujourd'hui les arts 182 à 188 du traité CE. 
l'association en stipulant notamment que «Les Etats membres appliquent à leurs échanges commerciaux avec les pays et territoires le régime qu'ils s'accordent entre eux» alors que «chaque pays et territoire applique à ses échanges commerciaux avec les Etats membres et les autres pays et territoires le régime qu'il applique à l'Etat européen avec lequel il entretient des relations particulières. L'article 133, paragraphe 1 prévoit que les importations en provenance des PTOM bénéficient à leur entrée dans les Etats membres de l'élimination totale des droits de douane. Cet article prévoit, dans son paragraphe 2, que les PTOM suppriment progressivement les droits de douane frappant les importations en provenance des Etats membres. Toutefois, le paragraphe 3 du même article prévoit que les pays et territoires peuvent recevoir des droits de douane qui répondent aux nécessités de leur développement et aux besoins de leur industrialisation ou qui, de caractère fiscal, ont pour but d'alimenter leur budget.

Dans l'affaire Pays-Bas c Conseil ${ }^{11}$ au point 49 de l'arrêt, la Cour de justice a également considéré que

(...) il convient de rappeler, à titre liminaire, la nature de l'association prévue par le traité pour les PTOM. Cette association fait l'objet d'un régime défini dans la quatrième partie du traité ( articles 131 à

136), de sorte que les dispositions générales du traité ne sont pas applicables aux PTOM sans référence expresse.

reprenant ainsi simplement la solution dégagée par la Cour dans l'arrêt Leplat.

Il n'en reste pas moins vrai qu'il est nécessaire de préciser cette solution. Tout d'abord les dispositions générales du droit communautaire ne sont pas applicables aux PTOM sans références expresses. De telles références peuvent figurer aussi bien dans les règles spéciales figurant dans le régime d'association lui-même ou dans des décisions du Conseil prises dans le cadre de ce régime, mais également directement dans d'autres dispositions du droit communautaire où il serait expréssément prévues qu'elles s'appliquent aux PTOM. Mais il n'en reste pas moins vrai qu'une disposition aussi essentielle que l'octroi par le traité CE de la citoyenneté de l'Union à toute personne possédant la nationalité d'un Etat membre et le fait que la Cour considère que dès lors aucune condition de résidence ou de domicile ne peut être exigée en droit communautaire pour que la personne concernée puisse bénéficier des droits que le traité lui reconnaît à ce titre. On ne peut être dès lors que déçu par la solution retenue par la Cour de justice, mais qui à notre avis ne concerne que l'exercice des droits de vote et d'éligibilité au Parlement européen.

\section{La question de la citoyenneté européenne}

Mais c'est la question de la citoyenneté européenne qui vient donner un tour nouveau à la question de l'association des PTOM à la Communauté. On sait en effet que l'article 17 du traité CE reconnaît comme «citoyen européen» toute personne qui possède la nationalité d'un Etat membre.

11 CJCE, arrêt du 12 novembre 2001, Royaume des Pays-Bas contre Conseil de l'Union européenne. Aff C110/97. Rec 2001, I-08763. 
Or au regard du droit constitutionnel français, les Polynésiens possèdent sans restriction aucune la nationalité française. Dès lors la solution devrait être claire et les Polynésiens être des citoyens européens à part entière avec toutes les conséquences qui sont attachées par le traité à un tel statut personnel. Dans une affaire relative aux droits de vote et d'éligibilité au Parlement européen, la Cour de Justice s'est récemment prononcée au sujet de l'application des articles 17 et suivants du traité CE.

Elle rappelle un point essentiel: l'article 17, paragraphe 1 deuxième phrase prévoit qu: «Est citoyen de l'Union toute personne ayant la nationalité d'un Etat membre». Il en résulte que le fait d'être domicilié ou d'avoir sa résidence dans un territoire faisant partie des PTOM, par exemple sur le territoire de la Polynésie française est sans incidence sur l'application de cette disposition. Ce qui importe en revanche est de posséder effectivement la nationalité d'un Etat membre de la Communauté. Il en résulte qu'un Polynésien, dès lors qu'il possède la nationalité française est de plein droit citoyen de l'Union et nous dit la Cour de justice qu'ils soient ou non résidant ou domicilié en Polynésie «peuvent invoquer les droits reconnus aux citoyens de l'Union dans la deuxième partie du traité». ${ }^{12}$

Cependant la Cour a admis que les Etats membres étaient cependant compétents, en l'état actuel de développement du droit communautaire, pour déterminer les conditions de mise en œuvre sur leur territoire de tels droits politiques. La Cour a considéré qu'il y avait lieu de répondre que: ${ }^{13}$

En l'état actuel du droit communautaire, si rien ne s'oppose à ce que les Etats membres définissent dans le respect du droit communautaire, les conditions du droit de vote et d'éligibilité aux élections au Parlement européen par référence au critère de résidence sur le territoire duquel sont organisées les élections, le principe d'égalité de traitement fait toutefois obstacle à ce que les critères choisis aient pour effet de traiter de manière différente des ressortissants se trouvant dans des situations comparables, sans que cette différence de traitement ne soit objectivement justifiée.

L'Acte final d'Amsterdam comportait une déclaration prévoyant en principe la révision du régime d'association des PTOM à la Communauté. On ne peut que constater la similitude des dispositions qui figurent dans le traité établissant une constitution pour l'Europe avec celles qui figurent positivement dans le traité $\mathrm{CE}$.

\section{La question de l'Euro}

Pour ce qui est de l'Union économique et monétaire, la situation des pays européens d'outre-mer n'est pas homogène. La France, Saint Pierre et Miquelon qui sont des PTOM au sens du droit communautaire ont l'euro comme unité monétaire alors que la Polynésie française au même titre que Wallis et Futuna ont le Franc Pacifique comme monnaie. Il avait été en effet prévu expréssément

12 Point 29 de l'arrêt rendu dans l'affaire C-300/04.

13 Point 61 de l'arrêt précité. 
lors du passage à l'euro que la France puisse conserver le privilège d'émettre sa propre monnaie dans ses territoires d'outre-mer si elle le désirait. Il semble que les élus de Nouvelle Calédonie aient quant à eux exprimé le désir d'adopter l'euro comme monnaie. Les autorités polynésiennes ont exprimé également un tel désir.

\section{B L'évolution du statut en cas d'évolution de la situation de la Polynésie française vis-à-vis de l'Etat membre avec lequel elle entretient des relations particulières}

Sur la question de savoir comment la liste des PTOM peut être modifiée, on remarquera que l'annexe II du traité CE n'a dans un premier temps pas fait l'objet de modifications malgré les changements de statut qui sont intervenus. C'est récemment le traité d'Amsterdam qui a procédé à la dernière mise à jour. D'une manière générale lorsqu'un PTOM accède à l'indépendance, il n'est plus possible de lui appliquer le régime communautaire de l'association des PTOM. Il est donc possible d'engager des négociations avec ce nouvel Etat tiers afin de lui permettre d'accéder le cas échéant à un nouveau statut communautaire, par exemple sa participation volontaire en tant que pays ACP à l'accord de Cotonou. On notera que le traité constitutionnel prévoit expréssément dans un article IV440, paragraphe 7, la possibilité de passer du statut de région ultrapériphérique à celui de PTOM ou inversement de celui de $\mathrm{PTOM}$ à celui de région ultrapériphérique. En droit positif, la décision d'association outre - mer, prévoir dans son article 61 la possibilité pour un PTOM d'accéder à l'indépendance et de ce fait son changement de statut communautaire. Ainsi le régime prévu par la décision en vigueur pourra continuer de s'appliquer provisoirement à ce pays dans des conditions qui seront fixées par le Conseil, cela pour éviter toute solution de continuité. En pratique des négociations seront ouvertes avec le pays afin de lui permettre d'accéder si les conditions sont remplies à la convention de Cotonou.

En guise de conclusion, on signalera qu'au mois d'octobre 2006, les seize dirigeants du Forum des îles du Pacifique se sont réunis aux îles Fidji pour leur $37^{\text {ème }}$ sommet. Papeete ne siège actuellement que comme observateur et souhaite pouvoir s'intégrer également dans cette organisation régionale. Comment concilier aide au développement et responsabilisation. Et la question qui demeure essentielle, la participation de la Nouvelle Calédonie et de la Polynésie française signifierait-elle à terme leur demande d'indépendance? Décidemment le statut de la Polynésie est au cœur du débat. 